\title{
Apoyo social percibido y riesgo de adicción a las redes sociales en estudiantes de una institución pública de Haquira
}

\author{
Perceived social support and risk of addiction to social networks in students of a \\ public institution in Haquira
}

Jemla Solis Baltazara' ${ }^{1}$, Alcides Quispe Mamani²

\section{RESUMEN}

Objetivo: determinar si existe relación significativa entre el apoyo social percibido y riesgo de adicción a las redes sociales. Metodología: diseño no experimental de corte transversal, tipo correlacional y de enfoque cuantitativo. La población fue de 153 estudiantes, utilizando un muestreo no probabilístico, con criterios de inclusión, estudiantes de 3 ro a 5 to grado, con conexión a internet y edades entre los 14 años a 18 años. Los instrumentos utilizados fueron la Escala Multidimensional de Apoyo Social Percibido (MMSP), alcanzando un índice de confianza de ,93 y una validez de $\mathrm{V}=, 99$. Respecto a la escala de Riesgo de Adicción a las Redes Sociales (C'ARS), alcanza un grado de confianza ,90 y validez de $V=, 1$; ambos instrumentos miden lo que deberían medir y de forma consistente. Resultados: existe correlación indirecta y altamente significativa (rho=-,303; $p<, 05$ ), así mismo respecto a los objetivos específicos de familia ( $r h o=-, 350 ; p<, 05)$ y Otros significativos (rho $=-, 247 ; p<, 05$ ), en cambio en el objetivo específico de amigos no se encontró relación indirecta (rho $=-, 143 ; p>, 05)$. Conclusión: los estudiantes que tengan mayor puntuación en apoyo social percibido de familia y otros significativos, menor puntuaciones tendrán en riesgo de adicción a redes sociales en cambio, en adolescentes que perciben apoyo de amigos no tendría el mismo efecto debido a que en las redes sociales pueden encontrar apoyo, también, tienen el riesgo de caer en un uso problemático.

Palabras clave: apoyo social percibido; riesgo de adicción; redes sociales; adolescentes

\begin{abstract}
Objective: to determine if there is a significant relationship between perceived social support and risk of addiction to social networks. Methodology: a non-experimental, cross-sectional, correlational type and quantitative approach design. 153 students were surveyed, using a non-probability sampling, with inclusion criteria, students from 3rd to 5th grade, with internet connection and ages between 14 and 18 years. The instruments used were the Multidimensional Scale of Perceived Social Support (MMSP), reaching a confidence index of .93 and a validity of $V=.99$. Regarding the scale of Risk of Addiction to Social Networks (C'ARS), it reaches a degree of confidence, 90 and validity of $V=, 1$; both instruments measure what they should measure and consistently. Results: there is an indirect and highly significant correlation (rho $=-.303 ; p<.05$ ), likewise with respect to the specific objectives of the family (rho $=-, 350 ; p<.05$ ) and other significant ones (rho $=-, 247 ; p<.05$ ), on the other hand, in the specific objective of friends, no indirect relationship was found (rho = -. 143; p> .05). Conclusion: students who have a higher score in perceived social support from family and other significant ones, lower scores will have at risk of addiction to social networks, on the other hand, in adolescents who perceive support from friends it would not have the same effect because in social networks they can find support, too, they run the risk of falling into problematic use.
\end{abstract}

Keywords: psychological well-being, psychological maturity, adolescence.

${ }^{1}$ Universidad Peruana Unión, Lima, Perú.

Orcid ID: 0000-0001-8380-874X

${ }^{2}$ Universidad Peruana Unión, Juliaca, Perú.

Orcid ID: 0000-0002-7404-7464 


\section{INTRODUCCIÓN}

En la actualidad, la sociedad en la que nos desarrollamos considera fundamental la formación académica y deja de lado aspectos primordiales como el desarrollo psicológico, los vínculos sociales y familiares, este aspecto está presente en especial durante la niñez y la adolescencia. Por ello, los esfuerzos de los padres y la sociedad se centran en el desempeño académico, los adolescentes que logran éxitos a nivel cognitivo son reconocidos, sin embargo, no se logra un desarrollo integral que repercute a lo largo de su vida (Ministerio de Educación, 2016). De todos los aspectos relacionados al desarrollo del ser humano, llama especial atención la influencia que tiene el entorno, es decir; la familia, los amigos y la escuela (Rodriguez, 2017). Por la coyuntura del confinamiento, los padres están cerca a los hijos, sin embargo, los estudiantes están expuestos a muchos riesgos, debido a que, el uso de redes sociales es frecuente, a si pueden fortalecer conexión emocional con los demás (Xu et al., 2020).

Al respecto, las estadísticas refieren que a nivel mundial alrededor del $59 \%$ de la población utiliza el internet activamente (Statista, 2020, como se citó en Arrivilga et al., 2020), fue incrementando un $7 \%$ al año 2019 , según reporta el informe que presenta anualmente la agencia global we are social (Machuca Rubio y Cabrera Duffaut, 2020). Se afirma que el número de usuarios de internet es de aproximadamente $4.021 \mathrm{mil}$ millones y 3.196 mil millones en redes sociales que utilizan de forma regular (Azizi et al., 2019; Castro, 2019), el $16 \%$ de la población mundial es de jóvenes, ellos ven común el uso de redes sociales en su día a día (Carnero Ortiz, 2020), pero, existen riesgos en redes sociales para la integridad física y mental de los estudiantes de entre 15 y 17 años, en algunos países, los niños menores a 15 años tienen la misma probabilidad de usar internet que los adultos mayores de 25 años, Estados Unidos, por ejemplo, el $92 \%$ de los adolescentes de entre 13 y 17 años manifiestan que están conectados todos los días (Machuca Rubio y Cabrera Duffaut, 2020). Por su parte la encuestadora chilena (Activa Research, 2019), encuestó a 4.929 padres sobre los hábitos de consumo y uso de internet de los niños, en 8 países de América, (Argentina, Canadá, Chile, Brasil, EEUU, Ecuador, México, Perú.) dando como resultado que la mayoría de los niños de la región inician en las redes sociales antes de los 13 años (Machuca Rubio y Cabrera Duffaut, 2020). Se descubrió también que 400 estudiantes universitarios, el $80 \%$ estaba suscrito a un plan de internet, el $53 \%$ revisaba el celular cada 15 minutos y las más frecuentes eran de carácter social $22 \%$ y académico $15 \%$ (Peñuela Espalza et al., 2014).

Respecto a la realidad nacional durante la cuarentena por el Covid-19 el 13.2 millones de peruanos conectados son usuarios de redes sociales, los más imprescindibles fueron Facebook, WhatsApp y YouTube (IPSOS PERÚ, 2020). Además de ello la compañía líder en la medición del mundo digital (ComScore, 2020) menciona que Perú es el país que tiene usuarios con más horas de consumo, llegando así a un promedio de 23 horas, durante el mes de julio, siendo la población masculina con mayor uso de redes sociales, pero, en el mes de mayo se ha incrementado notoriamente la participación de la población femenina (Adco, 2020).

En cuanto a la realidad de las regiones de la sierra, específicamente Apurímac la conexión a internet o el acceso a los teléfonos móviles es baja, tenemos las siguientes estadísticas según el Instituto Nacional de Estadística e Informática INEI menciona que del año 2007 al 2017 incrementó los hogares con conexión a internet del $6,8 \%$ a $28,0 \%$ de igual manera los hogares con teléfono móvil al menos un miembro del hogar del $42,9 \%$ a $83,8 \%$ a diferencia de aquellos hogares que son el $13,9 \%$ sin ningún acceso a tecnología de información y comunicación ( Instituto Nacional de Estadística e Informática (INEI), 2017). En la provincia de Cotabambas, distrito Haquira según una entrevista realizada a la Defensoría Municipal del Niño, Niña y Adolescente (DEMUNA, 2020), rebela que de los casos de conducta agresiva presentados el $70 \%$ es causada por el uso excesivo de celulares. De igual modo los directores de las instituciones educativas informaron que los estudiantes están presentando problemas y bajo rendimiento académico por el uso de redes sociales (Municipalidad Distrital de Haquira, 2020).

El uso desmedido de las redes sociales está asociado a problemas como el abandono escolar, insomnio, depresión, bajo rendimiento académico, entre otros (Arab y Díaz, 2015) y el apoyo social percibido del entorno no es adecuado, debido a esto, motiva a desarrollar 
el presente estudio sobre la relación entre el apoyo social percibido y el riesgo de adicción a redes sociales, también por la falta de estudios en el distrito de Haquira, específicamente en estudiantes de secundaria o adolescentes, se realiza este trabajo con el fin de estimular la investigación en el tema y proveer más conocimientos, así mismo servirá para estudios futuros más profundos y así promover que este problema reciba la importancia debida y se inicien proyectos, con el fin de brindar medidas de intervención oportuna hacia las familias, docentes y estudiantes.

Durante los últimos años el apoyo social es un tema de mayor interés en distintas disciplinas convirtiéndose en lo más estudiado del siglo XX (Alemán, 2013; Gracia et al., 2002). Remontándose desde la década de los sesenta con Jhon Bowly 1969; Cassel 1974 y Cobb 1976 quienes definieron al apoyo social como un efecto protector y equilibrio para momentos estresantes (Canas y Diaz, 2019; Cacsire, 2020). En los años '70 se desarrolló interés por conceptos de redes de apoyo, recursos del entorno (Abanto y Sachún, 2016), refiriéndose también a apoyo formal (instituciones) e informal (amigo, familia, compañeros, etc) siendo estos las fuentes más básicas y tradicionales (Alva Salinas, 2015). Ya en la década de los '80 House, Shumanker, Bonewell, Hobfoll y Stokes, mencionan que apoyo social no es solo la presencia de la persona, sino que debe dar una interacción emocional y social donde el otro se sienta querido (Citado de Canas y Díaz, 2019). El apoyo social es un intercambio de afecto positivo, interés emocional (Abanto y Sachun, 2016), conjunto de aportes que se recibe de tipo emocional, material, informacional y de compañía (García Ramos, 2021). Componente de interacción social con la familia, amigos y otros con quien se llega a tener contacto personal, emocional, instrumental o económica (Alva, 2015; Chan et al., 2019), puede ser también un factor protector o conductor de riesgo (Deng et al, 2021; Orcasita y Uribe, 2010). Las fuentes de apoyo son varias las que propone House 1981 son esposo/a, otros familiares, amigos, vecinos, jefes, compañeros, personas de servicio, grupos de autoayuda y profesionales de la salud (Thomson et al., 2017).

En cuanto al apoyo social percibido se define como la valoración cognitiva de estar vinculado a los demás (Cacsiere, 2020; Vasquez, 2020) y se utiliza para describir situaciones donde la asistencia brindada puede ayudar (Bareket et al, 2021), creer que el apoyo del otro está disponible proporcionando lo necesario (Burga Flores, 2020) y la calidad del apoyo (Wang et al., 2018) sirviendo como mediador hacia el bienestar psicológico (Lei et al., 2019; Malinauskas y Malinauskiene, 2020). Es cuando la persona se sienta respetada, apoyado, comprendido y satisfecho con la sociedad (Garcia Ramos, 2021). Las investigaciones indican que el apoyo social percibido está relacionado con las medidas de apoyo recibido (Gray et al, 2020), que se dividen en tres dimensiones, apoyo familiar, de amigos y otros significativos (Tarig et al, 2020; Vega,2020). Siendo la familia que ofrece a los adolescentes soporte en afrontamiento de situaciones personales (Vargas Dumian, 2018; Cacsiere Bautista, 2020), Shafter (2020) señalo que desde la mirada psicosocial las familias son proveedoras de apoyo social y emocional, ya que es un elemento básico de socialización con el exterior y otros sistemas sociales (Villafrade y Franco, 2016). Dai et al., (2021) refiere que el número de amigos está relacionado con el apoyo percibido, (Mosqueda et al., 2015) por su parte menciona que el grupo de amigos muestra influencias divergentes. Para Fernandez (2000) la institución educativa también es una red de apoyo (Orcasita et al, 2012).

Los adolescentes se caracterizan por cambios constantes donde experimentan situaciones diversas (Alcalde 2017) donde también están expuestos a diferentes riesgos uno de ellos son los teléfonos celulares ya que muchos de ellos viven pendientes a los destellos y vibraciones de un nuevo mensaje, debido a que es un medio donde también encuentran apoyo por medio de las redes sociales (Cargua Sevillano, 2019), la preocupación en los padres es la cantidad de tiempo que pasan en las redes sociales (Del Barrio y Ruiz, 2014).

Es por ello que en la presente investigación también se desarrollara el tema de las redes sociales siendo la tecnología pilar en la sociedad moderna (Arrvilga \& Extremmera, 2020). Se dio origen a la teoría de las redes sociales en los años 30 y 40, hasta ahora que es influenciado por diversas corrientes, en la década de los 70 y 80 esta teoría tuvo grandes avances (Liberato Reyes \& Polin Andrade, 2016). Las redes sociales o comunidad virtual fueron empleadas por primera vez por Howard Rheingold, mencionando como a un conjunto de personas, organizaciones o comunidades vinculadas a 
través de la comunicación en línea (Challco y Rodriguez, 2016).

Las redes sociales se definen como estructura compuesta de individuos de forma interpersonal, que incluyen sitios web, aplicaciones, que permite interacción entre una amplia gama de usuarios (Azizi et al., 2019).

La adicción a redes sociales, es considerada como un subtipo de adicción a internet, debido a que comparten característica enfocadas en el uso de sitios web o aplicaciones (Estrada y Gallegos, 2020), es una preocupación mental y asignación de tiempo al uso de redes que afecta otras actividades sociales (Azizi et al., 2019), que se asocia a problemas psicosociales, trastornos de personalidad, disminución de habilidades sociales (Arab y Díaz 2015; Arrivilga et al., 2020), es una dependencia a las plataformas virtuales debido a que los podemos tener en cualquier momento (Castro, 2019), por esta razón los más afectados son los adolescentes (Gomez y Sedin, 2014).

Hay un número creciente que sufren consecuencias negativas debido al uso de redes sociales (Hussan et al., 2020), donde también podemos identificar factores de riesgo como personales, familiares o sociales (Lachuman, 2020), la incorrecta implementación de las plataformas educativas, una supervisión inadecuada, estos representan un riesgo para la salud y la integridad del usuario (Carnero, 2020).

La investigación realizada por Ihm, J. (2018) nos demostró que el apoyo social está implicado en la adicción a los teléfonos móviles. A si mismo Castro (2019), menciona que el riesgo de adicción a redes sociales está asociado a la comunicación familiar (Hussan et al, 2020), y que un uso constante, genera influencia en las dinámicas familiares (Espinar y Gonzales, 2009). Los estudios realizados por (Estrada y Gallegos, 2020; Haro, 2018; Lachuman, 2020; Osorio, 2019; Vargas, 2018; Pinto, 2018) nos muestran una relación entre el funcionamiento familiar y adicción a redes sociales en los estudiantes. Podemos encontrar también que un uso excesivo de las tecnologías es relacionado a bajos niveles de apoyo percibido (Gonzales y Estevez, 2017), además de ello podemos referir que el apoyo social percibido es moderador entre el uso problemático de internet y la depresión (Gonzales, Estévez, 2017).
También tenemos la investigación realizada por Dai, $P$ et al., (2021) quien refiere que la gran cantidad de amigos se compensan a un escaso apoyo social percibido.

De las investigaciones revisadas podemos identificar que hay relación entre la familia y el riesgo de adicción a redes, que está condicionado por factores como familiar, social o ambiental (Sánchez et al, 2018).

Adicionalmente se observó que existen vacío en la literatura relacionados con el apoyo social percibido y riesgo de adicción a redes sociales en adolescentes, es así que la presente investigación pretende aportar a los estudios ya realizados de la relación que tiene los familiares, amigos y otros significativos en el riesgo de adicción a redes sociales, debido también a que en nuestra región el tema investigado es escaso.

Por lo anteriormente expuesto, se tiene como objetivo determinar la relación entre apoyo social percibido y riesgo de adicción redes sociales en adolescentes de una institución pública de Haquira. Los objetivos específicos procuran determinar la relación entre familia y riesgo de adicción a redes sociales, de la misma manera las demás dimensiones como: amigos y otros significativos.

\section{METODOLOGÍA}

En el presente estudio se optó por un diseño no experimental, puesto que el objetivo no era la modificación de variable alguna, si no el estudio típico del comportamiento de la variable apoyo social percibido y riesgo de adicción a redes sociales en un ambiente natural; es de tipo correlacional por que pretende estudiar la relación que existe entre dos variables (Apoyo social percibido y Riesgo de adicción a redes sociales). Es de enfoque cuantitativo por que el instrumento usado proporciona resultados numéricos las cuales será procesados bajo esta índole y de corte transversal porque se aplicará el instrumento una sola vez (Hernández, Fernández y Baptistas, 2014).

\section{Participantes}

Respecto a la población considerada en el estudio, se trabajó personas entre edades de 14 a 18 años, todos procedentes de la ciudad de 
Haquira; estas personas cursan estudios en un colegio estatal de la provincia Cotabambas de 3ro a 5to grado. Esta población alcanza los 500 sujetos. La muestra es de tipo no probabilístico, ya que trabajaran en base a criterios de inclusión y exclusión.

\section{Instrumentos}

El instrumento utilizado es la Escala Multidimensional de Apoyo Social Percibido cuya autoría de Zimet et al., 1988, adaptado al español por Landeta y Calvete, 2002 y adaptada al Perú por Juárez en el año 2018 posee 12 ítems destinados a identificar el nivel de apoyo social percibido, estos ítems están distribuidos en 3 dimensiones, Familia, Amigos y Otros significativos. Los valores de validez fluctúan en la dimensión familia con cuatro ítems entre 0.81 y 0.84 , en la dimensión amigos fue de 0.79 y 0.86 , también con 4 ítems, por último la dimensión otros significativos las cargas oscilan de 0.74 y 0.92 , y su grado de confiabilidad, en la dimensión familia es de 0.83 , en amigos 0.95 y en la dimensión otros significativos fue de 0.73 , lo que indica que no solo se trata de un instrumento con antecedentes en otros estudios, si no confiable y valido para el contexto muestral (Juárez, 2018).

En la tabla 1 se observa los índices de acuerdo a partir de la revisión del contenido por 5 jueces expertos, el índice alanzado en el constructo total, es de V - 0,99 este resultado indica que el instrumento es válido para su uso en investigación. Respecto a las dimensiones, Familia alcanza un nivel de V - 0, 98 este resultado indica que el instrumento es válido en contenido, respecto a la dimensión amigos esta obtiene un V - 1 este resultado indica que la dimensión es válida en cuanto su contenido, de la misma manera la dimensión otros significativos se obtiene un V - 1 que indica su validez en su contenido.

Tabla 1

Validez del instrumento apoyo social percibido

\begin{tabular}{llllll} 
Dimensiones & Claridad & Congruencia & Contexto & D. Constructo & V de Aiken \\
Familia & 0.95 & 0.95 & 1 & 1 & 0.98 \\
Amigos & 1 & 1 & 1 & 1 & 1 \\
Otros significativos & 1 & 1 & 1 & 1 & 1 \\
TOTAL & 0.98 & 0.98 & 1 & 1 & 0.99 \\
\hline
\end{tabular}

De igual modo se utilizó la escala de Riesgo de Adicción a las Redes Sociales, siendo sus siglas C'ARS, se construyó a partir de la teoría Cognitiva Conductual junto a diversos investigadores Young, 1997; Echeburua y Corral, 1994; Greenfield, 1999 y López, 2004 y adaptado por Vilca y Vallejos en el año 2015,

posee 43 ítems destinados a identificar el riesgo de adicción a las redes sociales, estos ítems están distribuidos en 7 dimensiones, Perdida de control con 10 ítems muestra un valor de validez 0.89 y confiabilidad de 0.91, síndrome de abstinencia con 7 ítems afirma una carga factorial de validez 0.86 y confiabilidad 0.85 , modificación del ánimo tiene 5 ítems con un valor de validez de 0.81 y confiabilidad 0.79 , interferencia en el ámbito social presenta 5 ítems con un valor de validez de 0.86 y confiabilidad 0.72 perdida de interés por otras actividades con 7 ítems tiene un valor de validez de 0.89 y confiabilidad 0.83 , dependencia con 5 ítems y un valor de validez de 0.76 y confiabilidad 0.72 y disminución del rendimiento académico de 4 ítems con un valor de validez de 0.86 y confiabilidad 0.86 . Lo que indica que el instrumento $s$ valido y confiable para el contexto muestral (Vilca y Vallejos, 2015).

En la tabla 3 se observa los índices de acuerdo a partir de la revisión del contenido en 5 jueces expertos, el índice alanzado en el constructo total, es de $\mathrm{V}-1$ este resultado indica que el instrumento es válido para su uso en investigación. Respecto a las dimensiones, alcanza un nivel de $\mathrm{V}-1$ este resultado indica que el instrumento es válido en cuanto a su contenido. 
Tabla 2

Confiabilidad del instrumento apoyo social percibido

\begin{tabular}{ll} 
Variable & Alfa \\
Apoyo social percibido 12 & .91 \\
\hline
\end{tabular}

De igual modo se utilizó la escala de Riesgo de Adicción a las Redes Sociales, siendo sus siglas C'ARS, se construyó a partir de la teoría Cognitiva Conductual junto a diversos investigadores Young, 1997; Echeburua y Corral, 1994; Greenfield, 1999 y López, 2004 y adaptado por Vilca y Vallejos en el año 2015, posee 43 ítems destinados a identificar el riesgo de adicción a las redes sociales, estos ítems están distribuidos en 7 dimensiones, Perdida de control con 10 ítems muestra un valor de validez 0.89 y confiabilidad de 0.91 , síndrome de abstinencia con 7 ítems afirma una carga factorial de validez 0.86 y confiabilidad 0.85 , modificación del ánimo tiene 5 ítems con un valor de validez de 0.81 y confiabilidad 0.79 , interferencia en el ámbito social presenta 5 ítems con un valor de validez de 0.86 y confiabilidad 0.72 perdida de interés por otras actividades con 7 ítems tiene un valor de validez de 0.89 y confiabilidad 0.83 , dependencia con 5 ítems y un valor de validez de 0.76 y confiabilidad 0.72 y disminución del rendimiento académico de 4 ítems con un valor de validez de 0.86 y confiabilidad 0.86 . Lo que indica que el instrumento $s$ valido y confiable para el contexto muestral (Vilca y Vallejos, 2015).

En la tabla 3 se observa los índices de acuerdo a partir de la revisión del contenido en 5 jueces expertos, el índice alanzado en el constructo total, es de $\mathrm{V}-1$ este resultado indica que el instrumento es válido para su uso en investigación. Respecto a las dimensiones, alcanza un nivel de $\mathrm{V}-1$ este resultado indica que el instrumento es válido en cuanto a su contenido.

Tabla 3

Validez del instrumento riesgo de adicción a las redes sociales

\begin{tabular}{llllll} 
Dimensiones & $\begin{array}{l}\text { Clari- } \\
\text { dad }\end{array}$ & $\begin{array}{l}\text { Con- } \\
\text { gruencia }\end{array}$ & $\begin{array}{l}\text { Contex- } \\
\text { to }\end{array}$ & $\begin{array}{l}\text { D. Cons- } \\
\text { tructo }\end{array}$ & $\begin{array}{l}\text { V de } \\
\text { Aiken }\end{array}$ \\
\hline Pérdida de control (LC) & 1 & 1 & 1 & 1 & 1 \\
Síndrome de abstinencia & 1 & 1 & 1 & 1 & 1 \\
Disminucion del rendimiento academico & 1 & 1 & 1 & 1 & 1 \\
Modificacion del estado de ánimo & 1 & 1 & 1 & 1 & 1 \\
Dependencia & 1 & 1 & 1 & 1 & 1 \\
Pérdida de interes en otras actividades & 1 & 1 & 1 & 1 & 1 \\
Conflictos en el ámbito social & 1 & 1 & 1 & 1 & 1 \\
TOTAL & 1 & 1 & 1 & 1 & 1 \\
\hline
\end{tabular}

Tabla 4

Confiabilidad del instrumento riesgo de adicción a las redes sociales

\begin{tabular}{lll} 
Variable & \multicolumn{1}{c}{ No Elementos $^{\circ}$} & Alfa \\
Riesgo de adicción a las redes sociales & 43 & 96 \\
\hline
\end{tabular}




\section{Análisis de datos}

Después de recolectar la información se utilizó el software SPSS en su versión 26 . Seguidamente se extrajeron los estadísticos de frecuencia respecto a ciertos datos sociodemográficos de la misma manera se obtiene tablas de frecuencia de personas que se sitúan en determinados niveles por cada variable de estudio, para determinar el tipo de distribución de las variables se hará uso de la prueba de bondad de ajuste por Kolmogorov Smirnov, los resultados demuestran que las variables no poseen una distribución normal, este último justifica el uso de la formula Rho de Spearman para determinar el índice de correlación entre las variables de la misma manera, esta fórmula se utiliza, para identificar la relación entre las dimensiones.

\section{RESULTADOS}

\section{Análisis sociodemográfico}

Se observa en la tabla 5 los resultados del análisis sociodemográfico aplicado a la población de estudio. En cuanto a la variable edad se observa que $32,7 \%$ tiene 15 años, seguido por un $31,4 \%$ que tiene 14 años, así mismo un $30,7 \%$ tiene 16 años y finalmente, un $5,2 \%$ tiene 17 años. En cuanto a la variable sexo, existe mayor proporción de mujeres $(50,3 \%)$ que varones $(49,7 \%)$. Finalmente se observa que un mayor porcentaje $(36,6)$ pertenece al quinto grado, así como el $35,9 \%$ al tercero y un $27,5 \%$ al cuarto grado.

Tabla 5

Características sociodemográficas de los participantes

\begin{tabular}{llll} 
Variable & Categoria & Frecuencia & Porcentaje \\
\hline Edad & 14 años & 48 & 31,4 \\
& 15 años & 50 & 32,7 \\
& 16 años & 47 & 30,7 \\
Sexo & 17 años & 8 & 5,2 \\
& Varón Mujer & 77 & 49,7 \\
Grado & & 76 & 50,3 \\
& Tercero & 55 & 35,9 \\
& Cuarto & 42 & 27,5 \\
\hline
\end{tabular}

\section{Análisis descriptivo}

En la tabla 6 se aprecia que la mayoría de los escolares presentan un apoyo social percibido moderado $(52.9 \%)$ existe un grupo importante que videncia un apoyo social percibido bajo $(26.8 \%)$. De manera similar se observa que el $29.4 \%$ presenta un alto riesgo de adicción a redes sociales y un $26.8 \%$ evidencia un bajo riesgo de adicción, también tenemos un grupo importante de estudiantes que presentan franca adicción a redes sociales (20.3\%).

Tabla 6

Niveles de apoyo social percibido y riesgo de adicción a redes sociales

\begin{tabular}{llll} 
Variable & Categoria & Frecuencia & Porcentaje \\
\hline Apoyo social percibido & Bajo & 41 & 26.8 \\
& Promedio & 81 & 52.9 \\
& Alto & 31 & 20.3 \\
& Total & 153 & 100.0 \\
\hline Riesgo de adicción a redes sociales & Bajo & 41 & 26.8 \\
& Medio & 36 & 23.5 \\
& Alto & 45 & 29.4 \\
& Adicción & 31 & 20.3 \\
& Total & 153 & 100.0 \\
\hline
\end{tabular}




\section{Análisis de normalidad}

Con el propósito de realizar los análisis comparativos y contrastar las hipótesis planteadas, se procedió a realizar primero la prueba de bondad de ajuste para precisar si las variables presentan una distribución normal. En ese sentido la tabla 7 presenta los resultados de la prueba de bondad de ajuste de KolmogorovSmirnov (K-S). Como se observa las variables en su mayoría no presenta distribución normal dado que el coeficiente obtenido $(\mathrm{K}-\mathrm{S})$ es significativo $(\mathrm{p}<0.05)$. Por tanto, para los análisis estadísticos correspondientes se empleará estadística no paramétrica.

Tabla 7

Análisis de normalidad

\begin{tabular}{llllll}
\hline Instrumentos & Variable & Media & D.E & K-S & P \\
\hline Apoyo social familiar & Escala global & 42.16 & 10.01 & 0.067 &, 094 \\
& Familia & 15.01 & 3.96 & 0.117 &, 000 \\
& Amigos & 12.8 & 4.29 & 0.133 &, 000 \\
& Otros significativos & 14.37 & 4.10 & 0.086 &, 007 \\
Riesgo de adicción a las redes sociales & Escala Global & 85.52 & 26.48 & 0.094 &, 002 \\
\hline${ }^{*} \mathrm{p}<0.05$ & & & & &
\end{tabular}

A continuación, se aprecia en la tabla 8 que existe una relación altamente significativa entre el nivel de apoyo social percibido y riesgo de adicción a redes sociales (rho=-,303; $p<.000$ ), es decir los participantes que tienen puntuaciones altas en apoyo social percibido presentan bajos puntajes en riesgo de adicción a redes sociales, respecto a las dimensiones de apoyo social percibido, se aprecia que familia (rho=-.,350; $p<.000$ ) y otros significativos (rho=-.247; $p<.002)$ presenta una relación altamente significativa al nivel de riesgo de adicción, es decir que los participantes que mayor nivel de apoyo social percibido de familias y otros significativos presentan bajo nivel de riesgo de adicción a redes sociales, sin embargo, la dimensión amigos no se relaciona significativamente con riesgo de adicción a redes sociales $(r h o=-.143 ; p<.078)$.

Tabla 8

Análisis de correlación entre las variables de estudio

\begin{tabular}{llll} 
& & \multicolumn{2}{l}{ Riesgo de adicción a redes sociales } \\
Instrumentos & Variable & Rho & $\mathrm{P}$ \\
Apoyo social percibido & Escala global &,$- 303^{* *}$ &, 000 \\
& Familia &,$- 350^{* *}$ &, 000 \\
& Amigos &,- 143 &, 078 \\
& Otros significativos &,$- 247^{* *}$ &, 002 \\
\hline
\end{tabular}

Nota: *La correlación es significativa al .01

\section{DISCUSIÓN}

El objetivo general de esta investigación, fue determinar la relación entre apoyo social percibido y riesgo de adicción a las redes sociales en estudiantes de una institución pública de Haquira, 2021. A partir del procesamiento estadístico se encontró que, si existe relación baja, indirecta y altamente significativa entre el apoyo social percibido y riesgo de adicción a redes sociales, es decir, mientras puntuaciones elevadas se observan en apoyo social percibido se observara menor riesgo de adicción a las redes sociales (rho=-,303; $p<, 05$ ). Estos resultados pueden ser comparados con Retuerto y Gutiérrez (2017); Lee, Kim, Choi y Yoo (2017) quienes mencionan en su investigación que un uso excesivo de las tecnologías está 
relacionado a bajos niveles de apoyo percibido y que también actúa como moderador en el uso problemático del internet. Estos resultados puede que se deban a que los que tienen grandes redes de apoyo, disminuye la adicción a los teléfonos inteligentes (Ihm, 2018) debido a que la adicción a redes sociales disminuye la percepción de apoyo social (Alimoradi, et al., 2019; Bai, et al., 2021) aquellas personas con apoyo social percibido y la calidad del mismo, reportan tendencias bajas al trastorno por uso de internet ( Jung, et al., 2019) que mayor uso de redes sociales, mayor es la necesidad de apoyo debido a que los adolescentes buscan constantemente atención y consideración (Veliz, 2020) y las personas perciben mayor apoyo con aquellos que comparten antecedentes o experiencias similares (Lee, 2020), siendo el apoyo social eficaz para el bienestar de uno, porque actúa como asistente de afrontamiento a distintas situaciones problemáticas (Agbaria, Q. y Abu, A. 2021), ayudando también en la integración social (Suría, 2017).

Respecto a los objetivos específicos en cuanto a la dimensión familia y riesgo de adicción a redes sociales existe una relación baja, indirecta y altamente significativa, es decir, mientras puntuaciones elevadas se observa en el apoyo social percibido en familia es menor el riesgo de adicción a las redes sociales (rho = -, 350; $\mathrm{p}<, 05)$. Estos hallazgos pueden ser comparados con Estrada y Gallegos (2020); Vilca y Vallejo (2015); Pinto (2018); Echeburua y Paz de Corral (2010) quienes refieren que inadecuado funcionamiento familiar está relacionado con altos niveles de adicción a redes sociales, debido a que el uso constante de redes sociales influye en las dinámicas familiares, por lo tanto el uso de redes puede ser utilizado para compensar ciertas carencias y malas relaciones (Gomez y Marin, 2017), donde no exista vínculo emocional, límites y reglas claras, mostrara mayor probabilidad de adicción a redes sociales (Lachuma, 2020), en aquellos usuarios de Facebook la satisfacción familiar es baja, debido a que la tecnología actúa como factor negativo en la vida familiar (lovu, Runcan, R., Runcan, P. y Andrioni, 2019), el ser humano necesita alcanzar satisfacción global en la vida, pero si es frustrada una o varias facetas, entonces puede centrar su atención en el mundo virtual (Echeurúa, 2012), el comportamiento parental positivo es un factor de protección contra el desarrollo de adicción a redes sociales (Yu y Lei, 2021; Cargua, 2020), así mismo la restricción reactiva y el monitoreo parental limita el riesgo de adicción a redes sociales (Yu y Luo, 2021; Arrivillaga, Rey y Extremera, 2020), siendo el apoyo familiar moderador entre la introversión y el uso compensatorio de redes sociales (Zhou, Li, Wang, Chen y Wang, K. , 2020), así mismo el apoyo social percibido en adictos para que el tratamiento tenga éxito necesita a su familiar, debido al potencial emocional que brinda la familia (Atadokht, Hjloo, Karimi y Narimani, 2014; Yang, Zhou y Xia, 2020).

En cuanto al objetivo específico de la dimensión otros significativos y riesgo de adicción a redes sociales existe una relación baja, indirecta y significativa, es decir, mientras puntuaciones elevadas se observa en el apoyo social percibido en otros significativos es menor el riesgo de adicción a las redes sociales (rho $=-$, 247; $p<, 05)$. Dichos hallazgos se pueden comparar con Zhang, et al., (2018) quien refiere que el apoyo de los compañeros de clase, colegas y el entorno están asociados al uso del internet, cuanto más fuerte es el apoyo percibido es menor la probabilidad de adicción a internet; debido a que el apoyo de los compañeros y el entorno escolar son cruciales en su desarrollo psicológico y su salud física y mental (Mitic, et al., 2021), siendo contribuyente en el bienestar del adolescente $(\mathrm{Bi}$, et al., 2021) tienen un efecto de prevención e intervención en diversos problemas (Griffin, et al., 2020), así mismo las relaciones positivas con los compañeros durante la adolescencia (Fernández - Zabala, A., Ramos - Díaz, E., Rodriguez - Fernández, A. y Nuñez, J. 2020) y el apoyo del maestro promueve bienestar mental (Guo, J., Liu, L., Zhao, B. y Wang, D. 2020) e influyen en el comportamiento (Jiménez, T., León, J., Martín-Albo, J., Lombas, A.S., ValdiviaSalas, S. y Estévez, E. 2021) y la autonomía del adolescente (Zhao, J. y Qin, Y. 2021) aquellos adolescentes que perciben a sus maestros como más comprensivos muestran más confianza entre sí (Miklikowska, M., Thijs, J. y Hjerm, M. 2019), un clima seguro en la escuela es un factor de protección (Jankowiak, B. et al., 2020), también dependiendo del rol adoptado pueden ofrecer apoyo o traer un conflicto en el adolescente (Núñez-Baila, M., Gómez-Aragón, A. y González-López, J. 2021) .Los jóvenes satisfacen las necesidades básicas y las necesidades de desarrollo de sus pares (Eccles y col., 1993; Deci y Ryan, 1995 citado en Zhao, J y Qin, Y. 2021), 
Por último, respecto a la dimensión amigos y riesgo de adicción a redes sociales no se encontró una relación significativa, es decir, mientras puntuaciones elevadas se observa en el apoyo social percibido en amigos existe riesgo de adicción a las redes sociales ( $r h o=-, 143 ; p>, 05$ ). Dicho hallazgo se puede comparar con Alrobai, et al. (2019) quien refiere que los adolescentes hacen uso de redes para tener popularidad, amigos, influencias con sus pares, sin embargo, pueden desarrollar uso problemático a redes sociales ya que resultaría ser un arma de doble cara. Esto puede que se deba a que el apoyo social percibido de familias reduce la adicción a los teléfonos móviles en cambio, el apoyo de los amigos no lo hace, debido a que buscan apoyo social en línea y esto puede provocar adicción (Zhao, et al., 2021) ya que los adolescentes prefieren establecer relaciones sociales a través de la tecnología, por lo tanto al enfrentase a una situación de interacción directa presentarían dificultades para expresarse (Mejía, Paz y Quinteros, 2014) y que el uso de redes sociales puede ser una forma de combatir ciertos efectos adversos del bajo apoyo social, debido a que tener uno o dos buenos amigos y conocer sus intereses similares puede ser más fácil en línea que en persona (Cole, et al., 2019) el número de amigos también es primordial ya que cuanto más reducido sea la cantidad de amigos mejor es el apoyo percibido, debido a que existirán lazos fuertes con ellos (Dai, et al., 2021), los amigos pueden influir positiva o negativamente en aspectos de salud (Rodriguez, 2017), el impacto de la sociedad actual influye en nuestra comunicación, debido a que el uso de las redes sociales proporciona apoyo social, logrando estar presentes virtualmente en la vida de familias amigos, recibiendo o dando consejos que ayuden al otro (Ortiz Peña, et al., 2019). Son visibles los beneficios y riesgos que implica las redes sociales y su influencia en el apoyo social percibido (Arab y Díaz, 2015).

\section{Declaración de financiamiento y de conflictos de interés:}

El estudio fue financiado por los autores, quienes declaran no tener conflictos de interés.

\section{Correspondencia}

Jemla Solis Baltazar

Correo electrónico:

jemasolis@upeu.edu.pe

\section{Alcides Quispe Mamani}

Correo electrónico:

alcides.quispe@gmail.com

\section{REFERENCIAS}

Abanto, E Abanto, E. P. y Sachún, L. (2016) Funcionamiento familiar, apoyo social y resiliencia en el adulto onologico. Hospital de alta complejidad virgen de la puerta - Trujillo [Tesis de licenciatura, Universidad Privada Antenor Orrego]. http://repositorio.upao.edu.pe/ bitstream/20.500.12759/2438/1/.PDF

Adco, P. (2020, 04 de diciembre). Estadísticas de redes sociales 2021 en Perú y Latinoamérica. https:// www.datatrust.pe/social- media/estadisticasredes-sociales/

Agbaria, Q. y Abu, A. (2021) Enfrentando el estrés durante el brote de coronavirus: la contribución de los cinco rasgos de personalidad y el apoyo social. Revista Internacional de Salud Mental y Adicciones. https://doi.org/10.1007/s11469-02100486-2

Alcalde, C. (2017) Apoyo socia percibido y resiliencia en Adolescentes embarazadas de centros de salud de Villa el Salvador. Acta Psicológica peruana, 2(2),235-250. http://revistas.autonoma. edu.pe/index.php/ACPP/article/view/77

Alemán, I. L. (2013). Desarrollo y validación del cuestionario de poyo social V.I.D.A. Vínculos interpersonales de apoyo. [Tesis doctoral, Universidad de las Palmas de Granada].https://accedacris.ulpgc.es/ bitstream/10553/11548/4/0696616_00000_0000. pdf

Alimoradi, Z., Chung, L., Imani, V., Griffits, M. y Pakpour, A. (2019) Adicción a las redes sociales y disfuncíon sexual entre las mujeres iranies: El papel mediador de la intimidad y el apoyo social. Revista de adicciones al comportamiento, 8(2), 318 - 325. DOI: 10.1556 / 2006.8.2019.24

Alrobai, A., Algashami, A., Dogan, H., Corner, T., Phalp, K. y Ali, R. (2019) Método COPE.er: Combatir la adicción digital a traves de grupos de apoyo de pares en línea. Revista internacional de investigacion ambiental y salud pública, 16(1162), 2- 39. doi: 10.3390 / ijerph16071162

Alva, A. (2015) Nivel de apoyo social percibido en la familia por la adolescente embarazada usuaria de un Hospital General de Lima Metropolitana [Tesis de Licenciatura, Universidad Mayor de San Marcos]. https://cybertesis.unmsm.edu.pe/ handle/20.500.12672/4939 
Arab, L. E. y Díaz, G. A. (2015). Impacto de las redes sociales e internet en la adolescencia: aspectos positivos y negativos. Revista Médica Clínica Las Condes, 26(1) 7-13. https://www.sciencedirect. com/science/article/pii/S0716864015000048

Arrivillaga, C., Rey, L. y Extremera, N. (2020). Perfil emocional de adolescentes en riesgo de un uso problemático de internet. Revista de Psicología Clínica con Niños y Adolescentes, 67(4), 2340-8340. https://www.researchgate.net/ publication/349142877

Atadokht, A., Hajloo, N., Karimi, M. y Narimani, M. (2015) El rol de la familia, expresion de la emociones y apoyo sicial perciido en la prediccion de riesgo de adicción. Revista de Adicciones, 4(1), 212 - 215. DOI: 10.5812/ijhrba.21250

Azizi, M. S., Soroush, A. y Khatony, A. (2019). La relación entre la adicción a las redes sociales y el rendimiento académico en estudiantes iraníes de ciencias médicas: un estudio transversal. BMC Psychology, 7-28. https://bmcpsychology. biomedcentral.com/articles/10.1186/s40359-0190305-0

Bai, J., Mo, K., Peng, Y., Hao, W., Qu, Y., Lei, X. y Yang, Y. (2021) La relación entre el uso de las redes sociales moviles y el bienestar subjetvo: el efecto mediador de la propensión al aburrimiento. Revista Fronteras en Psicología, 11(562849), 1 10. https://doi.org/10.3389/fpsyg.2020.568492

Bareket Bojmel, L., Shahar,G., Abu Kaf, S. y Margalit, M. (2021). Apoyo social percibido, soledad y esperanza durante la pandemia de COVID-19: probando un modelo de mediación en el Reino Unido, EE. UU. E Israel. Revista Británica de Psicología Clínica, 11111-12285. https:// onlinelibrary.wiley.com/

Burga Flores, M. P. (2020) Apoyo social percibido y resiliencia en internos drogodependientes en rehabilitación de la ciudad de Chiclayo [Tesis de Licenciatura, Universidad Señor de Sipán]. https://es.calameo.com/ books/0021424720dbf173098e7

Cacsire Bautista, K. J. (2020) Apoyo social percibido y su relación con la satisfacción familiar en niños y adolescentes [Tesis de Licenciatura, Universidad Nacional de San Agustín de Arequipa]. http://bibliotecas.unsa. edu.pe/bitstream/handle/UNSA/11269/PScabukj. pdf? sequence $=1$ \&isAllowed $=y$

Canas Bustios, M. D. y Diaz Huayna, L. M. (2019) "Resiliencia y apoyo social percibido en estudiantes de cuarto y quinto de secundaria del distrito de Ayaviri, Puno" [Tesis de Licenciatura, Universidad Católica San Pablo]. http://repositorio. ucsp.edu.pe/bitstream/UCSP/16017/1/.pdf
Cargua Sevillano, K. (2020) Estilos de crianza parental y su relación con el uso del internet en adolescentes de 14 a 16 años que asisten a la unidad educativa fiscal Calaclí en el periodo 2018-2019. [Tesis de Licenciatura, Universidad Central del Ecuador]. file:///C:/Users/DELL/ Desktop/cargua/estilos/crianza/sociales..pdf

Carnero Ortiz, O. E. (2020). Educación media superior y redes sociales en tiempos de pandemia. Acta Educativa, Revista Universidad Abierta, (58), 1-11. https://revista.universidadabierta.edu. $\mathrm{mx} / 2021 / 01 / 04 /$ educacion-media-superior-yredes-sociales

Castro Gonzales, C. (2019) Riesgo de adicción a las redes sociales y comunicación familiar en adolescentes de una Institución Educativa Privada de Lima [Tesis de Licenciatura, Universidad Peruana Unión]. https://repositorio.upeu.edu.pe/ handle/UPEU/2913

Challco Huaytalla, K.P. y Rodriguez Vega, S. Z. (2016) Riesgo de adicción a redes sociales, autoestima y autocontrol en estudiantes de 4to y 5to año del nivel secundario de una institución educativa pública de Lima Metropolitana este, 2015 [Tesis de Licenciatura, Universidad Peruana Unión]. https://repositorio.upeu.edu.pe/bitstream/handle/ UPEU/2056/

Chan, B., Goldman, E., Sarkar, U., Guzman, D., Critchfield, J., Saha, S. y Kushel, M. (2019). Alto apoyo social percibido y readmisiones hospitalarias en una población de mayor edad, multiétnica, con dominio limitado del inglés y red de seguridad. Revisit BMC Health Services Research, 334(2019), 2-9. https://bmchealthservres.biomedcentral.com/ articles/10.1186/s12913-019-4162-6

Cole, D., Nick, E., Varga, G., Smith, D., Zelkowitz, R., Ford, M. y Lédeczi, A. (2019) ¿Los aspectos del uso de twitter estan asociados con la reduccion de los síntomas depresivos? El papel moderador de la presencia en persona apoyo social. Revista Cyberpsicologia, comportamiento y social nortworking, 22(11), 692-698. DOI: 10.1089 / cyber.2019.0035

ComScore. (2020, 22 de junio). El crecimiento de las redes sociales en América Latina. https:// www.comscore.com/esl/Prensa-y-Eventos/ Presentaciones-y-libros-blancos/2011/The-RiseofSocial-Networking-in-Latin-America

Dai, P., Wang, N., Kong, L., Dong, X. y Tian, L. (2021) Una gran cantidad de amigos en línea y una alta frecuencia de interacción social se compensan mutuamente ' $\mathrm{s}$ escasez con respecto al apoyo social percibido. Revista Curr Psychol 1(2021)2-9 https://doi.org/10.1007/s12144-021-01458-4 
Del Barrio Fernández, A y Ruiz Fernández, I. (2014). Los adolescentes y el uso de las redes sociales. Revista INFAD de Psicología, 1(3), 571-576. https://revista.infad.eu/index.php//JODAEP/ issue/view/11

Deng, Y., Li, X., Liu, L. y Hong Chui, W. (2021). Intentos de suicidio y apoyo social percibido entre los consumidores de drogas chinos: el papel mediador de la autoestima y la depresión. Revista internacional de la Investigación ambiental y Salud Publica, 18, 208. https://www. mdpi.com/1660-4601/18/1/208

Echeburua, E. y Paz de Corral, G. (2010). Adicción a las nuevas tecnologías y a las redes sociales en jóvenes: un nuevo reto. Revista de Adicciones Vasco, 22(2), 91-96. https://dialnet.unirioja.es/ servlet/articulo?codigo $=3230123$

Espinar Ruiz, E. y Gonzalez Rio, M. J. (2009) Jóvenes en las redes sociales virtuales. Un análisis exploratorio de las diferencias de género. Revista Feminismos, 48(1989), 87-106. http://revistaredesrediris.es

Estrada Araos, G. y Gallegos Ramos, N. (2020). Funcionamiento familiar y adicción a redes sociales en estudiantes de educación secundaria Puerto Maldonado. Revista San Gregorio, 1(40), 101-117. https://dx.doi.org/10.36097/rsan. v1i40.1393

Fernández-Zabala, A., Ramos-Díaz, E., RodríguezFernández, A. y Núñez, J. (2020) Popularidad sociométrica, apoyo percibido entre pares y autoconcepto en la adolescencia. Revista fronteras en psicología 11 (594007), 1-10. doi: 10.3389/fpsyg.2020.594007

García Ramos, G. M. (2021) Apoyo social percibido y rendimiento académico en estudiantes de secundaria ante el covid - 19 [Tesis de Licenciatura, Universidad Católica de Santa María]. http://repositorio.ucsm.edu.pe/handle/ UCSM/33

Gomez Franco, F. y Sedin Gutierrez, J. C. (2014) Internet como refugio y escudo social: Usos problemáticos de la Red por jóvenes españoles. Revista científica de Educomunicacion, 43, 11343478. https://www.revistacomunicar.com/

Gómez Ferreira, K. K y Marin Quintero, J. S. (2017) Impacto que generan las redes sociales en la conducta del adolescente y en sus relaciones interpersonales en Iberoamérica los últimos 10 años [Tesis de licenciatura, Universidad Cooperativa de Colombia] https://repository.ucc. edu.co/bitstream/20.500.12494/747/1/.pdf

Gonzales Retuerto, N. y Estevez Gutierrez, A. (2017). El apoyo social percibido moderador entre el uso problemático de internet y la sintomatología depresiva en jóvenes adultos. Revista Health and Addictions Salud y Drogas, 17(1), 53-62. https:// www.redalyc.org/articulo.oa?id=83949782006

Gracia Fuster, E., Herrero Olaizola, J., \& Misitu Ochoa, G. (2002). Evaluación de recursos y estresores psicosociales en la comunidad. http://hdl.handle. net/10651/54767

Gray, I., Arora, T., Thomas, J., Saneh, A., Tohme, P. Y Abi Habib, R. (2020). El papel del apoyo social percibido en la depresión y el sueño durante la pandemia de COVID-19. Psychiatry Research, Revista Elsiever, 293(2020), 1-6. https://www. sciencedirect.com/science/article/abs/pii/ S0165178120331139?via\%3Dihub

Griffin, A., Sulkowski, M., Bámaca-Colbert, M. y Cleveland, H. (2020). Vidas sociales y afectivas diarias de los jóvenes sin hogar: ¿Cuál es el papel del apoyo social de maestros y compañeros?. Revista departamento de ayuda y servicios humanos USA, (2019) 77: 110-123. doi: 10.1016 / j.jsp.2019.09.004

Guo, J., Liu, L., Zhao, B. y Wang, D. (2020) Apoyo docente y bienestar mental en adolescentes chinos: El papel mediador de las emociones negativas y resiliencia. Revista fronteras en psicología, 10 (3081), 211. doi: 10.3389 / fpsyg.2019.03081

Haro Arrarte, E. (2018) Adiccion a redes sociales y funcionalidad familiar en estudiantes universitarios y egresados de Lima metropolitana. [Tesis de Licenciatura, Universidad San Ignacio de Loyola]. ...

Hernández Sampieri, R., y Mendoza Torres, C. (2018). Metodología de la investigación: Las rutas cuantitativa, cualitativa y mixta. México: McGrawHill Education. https://doi.org/10.1007/ s10899-018-9813-8

Hussan, Z., Wegmann, E., Yang, H. y Montag, C. (2020) Trastorno por uso de redes sociales y asociaciones con síntomas de depresión y ansiedad: una revisión sistemática de investigaciones recientes en China. Revista Fronteras en Psicología, 11(211), 1-11. https://www.frontiersin.org/articles/10.3389/ fpsyg.2020.00211/full

Instituto Nacional de Estadística e Informática (INEI). (2017). Hogares según coberturas de las tecnologías de información y comunicación. Perú: Perfil Sociodemográfico, 392-409.

lovu, M., Runcan, R., Runcan, P. y Andrioni, F. (2020) Asociación entre uso de faceook, depresión y satisfaccion familiar: un estudi trnasversal de la juventud rumana. Revista Iran Public Health, 49(11), 2111 - 2119. http://ijph.tums.ac.ir (2020, 23 de junio). Uso de las redes sociales 
entre peruanos conectados de $2020 \mathrm{https} / /$ www.ipsos.com/sites/default/files/ct/publication/ documents/2020-08/redes_sociales_2020_ v3_6ago_20.pdf

Jankowia, B., Jaskulska, S., Sanz-Barbero, B., Ayala, A., Pyzalski, P., Bowes, N., De Claire, K., Neves, S., Topa, J., Rodríguez-Blázquez, C., DavóBlanes, M., Rosati, N., Cinque, M., Mocanu, V., Ioan, B., Chmura-Rutkowska, I., Waszynska, K. y Vives-Cases, C. (2020) El papel del apoyo social escolar y el clima social escolar en la prevención de la victimización por violencia en el noviazgo entre los adolescentes en Europa. Revista internacional de investigación ambiental y salud pública. (17), 8935, 213. https://www.mdpi.com/ journal/ijerph

Jiménez, T., León, J., Martín-Albo, J., Lombas, A.S., Valdivia-Salas, S. y Estévez, E. (2021) Vínculos transaccionales entre el apoyo de los maestros y los adolescentes, la relación y la agresión en la escuela: una triple ola.Estudio longitudinal. Revista internacional de investigacion ambiental y salud pública, 18, (436), 2-19. https://doi. org/10.3390/ijerph180204

Jung, S., Sindermann, C., Li, M., Wernicke, J., Quan, L., Ko, H. y Montag, C. (2019) Estilos de afrontamiento relacionados con la ansiedad, apoyo social y trastorno por uso de internet. Revista Fronteras en Psicología, 10(640), 1 13. https://www.frontiersin.org/article/10.3389/ fpsyt.2019.00640/full.

Lachuma Huansi, U. R. (2020) Funcionamiento Familiar y Adicción a Redes Sociales en Estudiantes del Nivel Secundario de una Institución Educativa Pública de Lima Este [Tesis de Licenciatura, Universidad Peruana Unión]. https://repositorio.upeu. edu.pe/bitstream/handle/UPEU/3357/_2020. pdf? sequence $=4 \&$ isAllowed $=y$

Lee, S., Kim, H., Choi, H. y Yoo, Y. (2017) Adiccion a los teléfonos inteligentes y competencia interpersonal de estudiantes de enfermeria. Revista Iran Public Health, 47(3), 342 - 349. http://ijph.tums.ac.ir

Lei, J., Ashwin, C., Brosnan, M. y Russell, A. (2019) Desarrollo de una herramienta en línea para medir la estructura de las redes sociales y el apoyo social percibido entre los estudiantes autistas en la educación superior: un estudio de viabilidad. Revista de autismo y trastornos del desarrollo, 49, 3526-3542. https://link.springer. com/article/10.1007\%2Fs10803-019-04070-5

Liberato Reyes, I. y Polin Andrade, J. J. (2016) Riesgo en adicciones a redes sociales y adaptación de conducta en estudiantes de 1 er ciclo de una universidad privada de Lima Este [Tesis de Licenciatura, Universidad
Peruana Unión]. https://repositorio.upeu.edu. pe/bitstream/handle/UPEU/479/Irma_2016. pdf? sequence=3\&isAllowed $=y$

Malinauskas, R. y Malinauskiene, V. (2020) La relación entre la inteligencia emocional y el bienestar psicológico entre estudiantes universitarios varones: el papel mediador del apoyo social percibido y el estrés percibido. Revista Internacional de Investigación ambiental y salud pública, 17(5) 1-16. https://www.mdpi. com/1660-4601/17/5/1605

Mejía, G., Paz, J. y Quinteros, D. Adicción a facebook y habilidades sociales en estudantes de una universidad privada de Lima. Revista cientifica de ciencias de la salud, 7(1), 7- 15. https://www. academia.edu/34898967/PB_1_?from=cover_page

Miklikowska, M., Thijs, J. y Hjerm, M. (2019) El impacto del apoyo percibido por los maestros en las actitudes antiinmigrantes desde el principio hasta el final de la adolescencia. Revista de juventud y adolescencia (2019) 48: 1175-1189 https://doi. org/10.1007/s10964-019-00990-8 Ministerio de Educacion. (2016, 25 de agosto). Ministerio de Educación: http://www.minedu.gob.pe/politicas/ aprendizajes/

Mitic, M., Woodcock, K., Amering, M., Krammer, I., Stieh, K., Zehetmayer, S. y Schrank, B. (2021) Hacia un modelo integrado de relaciones de apoyo entre pares en la adolescencia temprana: una revision sistematica y exploratoria Metaanalisis. Revista Fronteras en Psicología, 12(589403), 1-28. https://doi.org/10.3389/fpsyg.2021.589403

Mosqueda Diaz, A., Mendoza Parra, S., Jofre Aravena, V. y Barriga, O. A. (2015) Validez y confiabilidad de una escala de apoyo social percibido en población adolescente. Revista Electrónica Trimestral Enfermería Global, 39(16) 125- 135. https://scielo.isciii.es/scielo.php?script=sci_ arttext\&pid=S169561412015000300006

Municipalidad Distrital de Haquira. (27 de diciembre de 2020). Municipalidad Distrital de Haquira, https://www.peru.gob.pe/Nuevo_Portal_ Municipal/portales/Municipalidades/307/ entidad/pmmunicipali dad_tematicos.asp?cod_ tema $=81680$

Núñez-Baila, M., Gómez-Aragón, A. y GonzálezLópez, J. (2021) Apoyo social e integración de grupos de pares de adolescentes con diabetes. Revista internacional de invvestigacion ambiental y salud pública, (18) 2064, 1-14, https://doi. org/10.3390/ijerph18042064

Orcasita Pineda, L. T. y Uribe Rodriguez, A. F (2010) La importancia del apoyo social en el bienestar de los adolescentes. Revista Psychology: Avances de la Disciplina. 4(2), 69-82. https://dialnet. unirioja.es/servlet/articulo?codigo $=4924034$ 
Orcasita, L.T., Uribe, A.F., Castellanos, L. P. y Gutierrez Rodriguez, M. (2012) Apoyo social y conductas sexuales de riesgo en adolescentes del municipio de Lebrija-Santander. Revista de Psicología, 30(2), 373-406. https:// scielo.conicyt.cl/scielo.php?script $=$ sci_ arttext\&pid=S0718-48082019000300211

Ortiz Peña, M., Navarro Oliva, E., Echevarría Cerda, F., Guzmán Facundo, F., Pacheco Pérez, A. y Rodriguez, L. (2019) Apoyo social percibido a traves de facebook, familia y amigos y el consumo de alcohol en adolescentes. Revista Apoyo y Adicciones Salud y Drogas, 19(2), 103-110

Osorio Llaja, E. (2019) Relaciones intrafamiliares y apoyo social percibido en estudiantes universitarios. [Tesis de Segunda Especialidad, Universidad Nacional Federico Villarreal]. http:// repositorio.unfv.edu.pe/handle/UNFV/3116.

Peñuela, N., Paternina del Río, J. , Moreno, D. , Camacho, L. , Acosta, L. y De León, L. (2014) El uso de los smartphones y las relaciones interpersonales de los jovenes univversitarios en la ciudad de Barranquilla (Colombia). Revista salud Uninorte Barranquilla, 30(3), 335 - 346. ttp://dx.doi.org/10.14482/sun.30.1.4309

Pinto Benavente, A. (2018) Funcionamiento familiar y adicción a las redes sociales en estudiantes de tercero, cuarto y quinto de secundaria [Tesis de licenciatura, Universidad Nacional Mayor de San Marcos]. https://cybertesis.unmsm.edu. pe/bitstream/handle/20.500.12672/9785/Pinto_ ba.pdf?sequence=1\&isAllo wed=y

Rodriguez Molinero, L. (2017). El adolescente y su entorno: familia, amigos, escuela y medios. Revista pediatria integral, 21(4), 261 - 269. file:///C:/Users/ DELL/Desktop/adolescente /y/ su/entorno.pdf

Sánchez, L. Benito, R. Serrano, A. Benavent, R. y Bueno, F. (2018). Programa de prevención del uso problemático de internet y redes sociales. "Clickeando." Concejalía de Sanidad, Salud y Deportes. Valencia. https://www.cendocbogani. org/es/f-pmd/2282/1/anyo-0/8/programa-deprevencion-del-usoproblematico-de-internet-yredes-sociales-clickeando

Tariq, A., Bihai, T., Abbas, N., Ali, S., Yao, W. y Imran, M. (2020) Papel del apoyo social percibido en la asociación entre discapacidad física y síntomas de depresión en personas mayores de Pakistán. Revista Internacional de Investigacion ambiental y Salud Publica, 18(9) ,2-14. https://www.mdpi. com/journal/ijerph

Vargas Dumian, C. E. (2018) Funcionamiento familiar y apoyo social percibido en estudiantes de una universidad pública de Lima [Tesis de Maestría, Universidad Nacional Federico Villarreal]. http:// repositorio.unfv.edu.pe/handle/UNFV/2776
Vásquez Vega, E. J. (2020) "Apoyo social percibido y violencia escolar en adolescentes de educación secundaria" [ Tesis de Doctor, Universidad Nacional Federico Villarreal]. http://repositorio. unfv.edu.pe/bitstream/handle/UNFV/4125/ V\%DOCTORADO.pdf? sequence $=1$ \& is All owed=y

Vega de Salas, S. J. (2020) Apoyo social percibido y resiliencia en estudiantes becados de una universidad privada de Lima Metropolitana [Tesis de Bachillerato, Universidad San Ignacio de Loyola]. http://repositorio.usil.edu.pe/bitstream/ USIL/10090/1/2020_Vega\%20De\%20Salas.pdf

Veliz Martinez, J. (2021) Autoestima y apoyo social percibido en universitarios que hacen uso de redes sociales. [Tesis de licenciatura, Universidad Peruana de Ciencias Aplicadas]. http://hdl.handle. net/10757/653137

Vilca, L., \& Vallejos, M. (2015). Construcción de la Escala de Riesgo de Adicción a las Redes Sociales (Cr.ARS). Revista ELSEVIER, 190 - 198 https://dl.acm.org/doi/abs/10.1016/j. chb.2015.01.049

Villafrade Monroy, L. A. y Franco Ramirez, C. M. (2016) La familia como red de apoyo social en estudiantes universitarios que ingresan a primer semestre. Espiral, Revista de Docencia e Investigación, 6(2), 79-90. https://www.bing.com/ search?q=Villafrade+Monroy\%2C+L.+A.+y+ Franco + Ramirez\%2C+C.+M.+7990.\&cvid $=d 6 a 6663163 a$ 346f29161e77fb3d44510\&aqs=edge..69i57676j0j $4 \& F O R M=A N A B 01 \& P C=U 531$

Wang, J., Mann, F., Lloyd Evans, B., Ma, R. y Johnson, S. (2018) Asociaciones entre la soledad y el apoyo social percibido y los resultados de los problemas de salud mental: una revisión sistemática. Revista BMC Psychiatry, 18(156), 2-16. https://bmcpsychiatry.biomedcentral.com/ articles/10.1186/s12888-0181736-5\#citeas

Xu, J., Ou, J., Luo, S., Wang, Z., Chang, E., Novak, C., Shen, J., Zheng, S. y Wang, Y. (2020) El apoyo social percibido protege a las personas solitarias contra la ansiedad COVID-19: un estudio longitudinal de tres ondas en China. Revista Fronteras en Psicología, 11(20), 2-12. https://www.frontiersin.org/articles/10.3389/ fpsyg.2020.566965/full

Yang, C., Zhou, T. y Xia, M. (2020) ¿Cómo la resilienia promuevev la salud mental de los pacientes con trastornos por consumo dde sustancias DSM5 ? Los roles de mediación del afecto positivo, autoestima y poyo social perciiy el apoyo social percibido. Revista fronteras de psicologia, 11(588968)1-12. https://www.frontiersin.org/ articles/10.3389/fpsyt.2020.588968/full 
Yu, L. y Lei Shek, D. (2021) Atributor positivos del desarrollo juvenil y la paternidad como fatores protectores contra la adiccion a las redes sociales de los adolescentes en Hong y Kong. Fronteras en psicología. 9(649232), 1- 13. https://doi. org/10.3389/fped.2021.649232.

Yu, L. y Luo, T. (2021) Adicción a las redes sociales entre estudiantes de la univversidad de Hong y Kong; sus consecuenias para la salud y relaciones con los comportamientos de los padres. Revista Fronteras en psicología, 8(555990) 1- 11. https:// doi.org/10.3389/fpubh.2020.555990

Zhang, S., Tian, Y., Sui, Y., Zhang, D., Shi, J., Wang, P., Meng, W. y Si, Y. (2018) Relaciones entre el apoyo social, la soledad y la adiccion a internet en estudiantes chinos de educacion superior: una perspectiva longitudinal analisis rezagado cruzado. Revista fronteras en psicología, 9(1707), 1-13. https://doi.org/10.3389/fpsyg.2018.01707

Zhao, C., Xu, H., Lai, X., Yang, X., Tu, X., Ding, N., Lv, Y. y Zhang, G. (2021) Efectos del apoyo social en línea y el apoyo social percibido en la relaión entre el estrés percibido y el uso problemático de telefonos inteligentes entre estudiantes chinos. 3(14), 529-539. https://doi.org/10.2147/PRBM. S302551

Zhao, J. y Qin, Y. (2021) Apoyo a la autonomía percibida del maestro y aprendizaje profundo de los estudiantes: el papel mediador de la autoeficacia y el papel moderador del apoyo percibido de los compañeros. Revista fronteras en psicología, 652796 (12), 2-11. doi: 10.3389 / fpsyg.2021.652796.

Zhou, Li, Wang, Chen y Wong (2020) Uso compensatorio del sitio de redes sociales, apoyo familiar y depresion entre estudiantes universitarios de primer año: estudio de panel de tres oleadas. Revista Revista JMIR Publicaciones, 22(9), 1438-187. https://www.ncbi.nlm.nih.gov/ pmc/articles/PMC7495252/?report=printable

Recibido: 16/07/2021

Aceptado: 19/11/2021 